\title{
A micromagnetic study: lateral size dependence of the macroscopic properties of rectangular parallelepiped Cobalt-ferrite nanoferromagnetic
}

\author{
Nur Aji Wibowo ${ }^{1,3 *}$, Susatyo Pranoto ${ }^{1}$, Cucun Alep Riyanto ${ }^{2,3}$, Andreas \\ Setiawan ${ }^{1,3}$ \\ ${ }^{1}$ Department of Physics, ${ }^{2}$ Department of Chemistry, \\ ${ }^{3}$ Study Center for Multidisciplinary Applied Research and Technology, \\ Universitas Kristen Satya Wacana, Indonesia, Jalan Diponegoro 52-60 Salatiga \\ *Corresponding author’s e-mail: nur.wibowo@uksw.edu
}

Received 15 December 2019, Revised 8 February 2020, Published 29 March 2020

\begin{abstract}
The purpose of this study is to provide systematic information through micromagnetic simulations related to the impact of particle size on the magnetic characteristics of Cobalt-ferrite MNP. The micromagnetic computations performed were based on LLG equation. The MNPs sample was simulated in the form of a rectangular parallelepiped with a thickness of $20 \mathrm{~nm}$ and square surface with lateral length varies from 10 to $80 \mathrm{~nm}$ at an interval of $10 \mathrm{~nm}$. The results of this study indicate that the size changes in Cobalt-ferrite MNP have a significant impact on various magnetic properties, such as the magnitude of the barrier energy, coercive and nucleation fields, magnetization rate, magnetization curve profile, and magnetization mode. Cobalt-ferrite MNP with a size of $10 \mathrm{~nm}$ shows a single domain with a relatively short magnetization reversal time and high coercive field.
\end{abstract}

Keyword : Cobalt-ferrite, micromagnetic, nanoparticles.

\section{Introduction}

The nanotechnology industry is growing rapidly and promises significant changes in various fields in the present, such as aerospace engineering, environmental improvement, health, and nano-electronics (Amiri \& Shokrollahi, 2013). Magnetic nanoparticles (MNPs) have numerous unique properties and offer exciting new options in many applications. These properties of MNPs are categorized into three, namely macroscopic, intrinsic magnetic and physical structure properties. Macroscopic properties include coercive field, nucleation field, and energy density. Intrinsic properties include magnetization, magneto-crystalline anisotropy, and exchange constant. Physical structure properties are characterized by particle size and shape. These properties determine the performance of MNPs, both in terms of the intensity of magnetic field it can produce, and the sensitivity of its response to external magnetic field (Fischbacher et al., 2018). These properties can be controlled through modification 
of its synthesis method (Ganachari et al., 2017; Houshiar et al., 2014; Purnama et al., 2015). The one of the fundamental aspects of MNPs in its application is its particle size. In terms of size, potential MNPs are those that have strong magnetic properties even though they are extremely small.

MNPs which in the last two decades has been considered by many researchers to be used is Cobalt-ferrite $\left(\mathrm{CoFe}_{2} \mathrm{O}_{4}\right)$. The unique physical and mechanical characteristics of Cobalt and Ferrite are the key factors in the development of nanotechnology. Cobaltferrite is mechanically durable (Srinivasan et al., 2018) and has strong coercivity despite its small size (Limaye et al., 2009), and its Curie temperature is quite high (Mohamed, 2010). This material is also chemically stable, electrical insulating, and easy to synthesize (Amiri \& Shokrollahi, 2013).

The micro structure of Cobalt-ferrite MNP greatly influences its magnetization reversal behavior. This behavior determines the important extrinsic properties of Cobalt-ferrite MNP. In the effort to improve the performance of Cobalt-ferrite MNP, it needs not only research regarding synthesis and characterization processes, but also computation, specifically micromagnetic computation. This micromagnetic numerical simulation is one of the pillars in the development of material physics, in addition to experiments and theoretical studies (Bedanta et al., 2013). In addition to being used to understand its magnetization reversal mechanism well (Bance et al., 2015), micromagnetic computation method can also be used to study the effects of microstructure on the magnetic properties of a material, so that the simulation results can be used as a reference for MNPs development (Fischbacher et al., 2018). However, there are only few micromagnetic studies of nano-Cobalt-ferrite.

Before realizing nanoparticles, including Cobalt-ferrite MNP, a comprehensive understanding of various aspects is needed to encourage extensive fundamental research (Guo et al., 2018). However, fundamental research related to magnetic characteristics is rarely noticed (Ghazanfari et al., 2016). Therefore, this work will complement fundamental information related to the magnetic features of Cobalt-ferrite MNP. The purpose of this study is to provide a systematic basic understanding through micromagnetic simulations related to the magnetic characteristics of Cobalt-ferrite MNP. In particular, we will discuss the relationship between changes in particle size and magnetic features of Cobalt-ferrite MNP. The characteristics studied focus only on the critical properties in applications, such as energy density, material coercivity, nucleation field, magnetization reversal rate, and magnetization behavior.

\section{Numerical Method}

In this micromagnetic study, the MNPs was simulated in the form of a rectangular parallelepiped with a thickness, $D$, of $20 \mathrm{~nm}$ and square surface with lateral length of $L$. This sample consisted of elements that were also rectangular parallelepiped with a thickness of $D$ and square surface with lateral length of $l$, where $L=n l$ with $n$ is the number of elements. To make it smaller than the width of the domain wall (Shir, 1978), $l$ was set to be $3.3 \mathrm{~nm}$. 


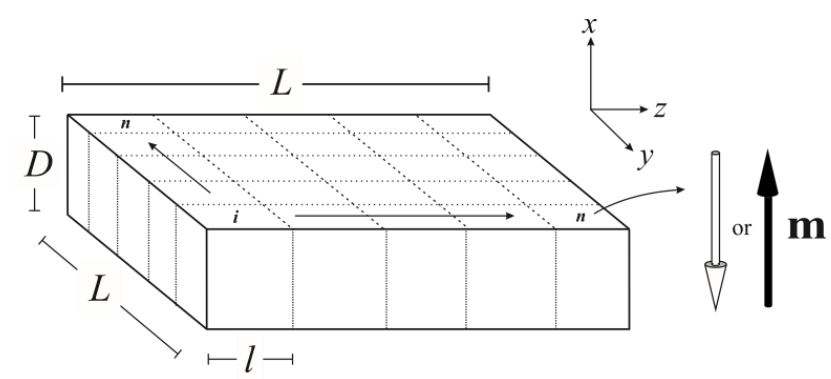

Figure 1. Magnetic nanoparticle model.

The thin layer consisted of $n^{2}$ elements. In this study, $n$ was varied from $3,6,9,12$, $15,18,21$, to 24 , so that the surface areas of the layers were $10 \times 10 \mathrm{~nm}^{2}, 20 \times 20 \mathrm{~nm}^{2}$, $30 \times 30 \mathrm{~nm}^{2}, 40 \times 40 \mathrm{~nm}^{2}, 50 \times 50 \mathrm{~nm}^{2}, 60 \times 60 \mathrm{~nm}^{2}, 70 \times 70 \mathrm{~nm}^{2}$, and $80 \times 80 \mathrm{~nm}^{2}$. The size of $L$ was designed in the order of tens of nanometers. In that order, the effective surface becomes wider (Akbarzadeh, 2012). Illustration of MNPs and its constituent elements is shown in Figure 1.

The investigated MNPs have the characteristics of Cobalt-ferrite with the chemical structure of $\mathrm{CoFe}_{2} \mathrm{O}_{4}$. According to the experiment previously conducted by Houshiar et al., Amiri et al., and Rondinone et al., the magnetic parameters used in this simulation were magnetic saturation, $4 \pi M_{\mathrm{s}}$, of 3735 Gauss (Houshiar et al., 2014) with magnetic anisotropy, $K$, of $1.90 \times 10^{6} \mathrm{erg} / \mathrm{cc}$ (Amiri \& Shokrollahi, 2013) and $2.23 \times 10^{6} \mathrm{erg} / \mathrm{cc}$ (Rondinone et al., 2000). The easy axis of the MNPs was set in the direction of the $x$ axis, so that the magnetization direction was saturated perpendicularly to the surface of the layer. Each element contains magnetic moment, $\mathbf{m}$. The magnetization of the MNPs is the resultant value of the magnetic moment of its constituent elements $\left(\mathbf{M}=\left(n^{2} / V\right)\right.$ m). Other material parameters used in this simulation included Curie temperature of 373 $\mathrm{K}$, exchange interaction constant of adjacent elements of $1.0 \times 10^{-7} \mathrm{erg} / \mathrm{cm}$, damping Gilbert constant of 0.3 , and gyromagnetic ratio of $1.76 \times 10^{7} \mathrm{Oe}^{-1} \cdot \mathrm{s}^{-1}$. In the computation, the integration step used was 0.25 ps.

To determine the impact of the size of Cobalt-ferrite MNP on its magnetic properties and reversal behavior, a micromagnetic computation approach was used. Computations were performed using a micromagnetic software by Konishi et al. (Purnama et al., 2009; Wibowo et al., 2014; Purnama et al., 2007; Wibowo et al., 2019; Wibowo et al., 2019). The basis of the work of this device is the Landau-Lifshift-Gilbert (LLG) equation (Azizah et al., 2017; Suess et al., 2002). In this simulation, the MNPs model, which was initially magnetized in the direction of the $x$-axis $(+)$, was conditioned at room temperature $(\mathrm{T}=298 \mathrm{~K}$ ) while being induced by an external magnetic field towards the $x$-axis (-), so that the sample part exposed to the field was only the square surface with an area of $L^{2}$. The intensity of this inducing magnetic field was increased linearly from 0 to 2 Tesla for $2.5 \mathrm{~ns}$. 

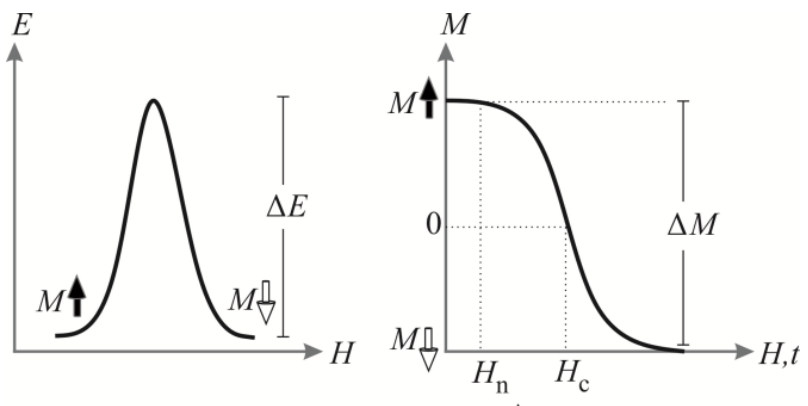

$\vdash \Delta t \longrightarrow$

Figure 2. Basic profile of, (a). Barrier energy, (b). Nucleation and coercive field.

Some of the magnetic properties of Cobalt-ferrite MNP studied were barrier energy $(\Delta E)$, nucleation field $\left(H_{\mathrm{n}}\right)$, coercive field $\left(H_{\mathrm{c}}\right)$, and reversal rate $(\Delta M / \Delta t)$. The barrier energy was measured from the height of the energy densities of the sample during the induction using an external magnetic field (Figure 2(a)). This barrier separated two stable states of magnetization. The magnetic field needed to start the magnetization change is called the nucleation field. On the other hand, the field required to reverse half of the total magnetization is called the coercive field. The nucleation and coercive fields were determined from the formed magnetization curve while the sample was being induced, as shown in Figure 2(b) (Richter \& Harkness, 2006).

\section{Result and Discussion}

The total energy of the MNPs is determined by several forms of energy, namely Zeeman energy, anisotropic energy, exchange energy, and magnetostatic energy (Schrefl et al., 2006). Zeeman's energy appears because of the interaction of the external magnetic field with the magnetic moment of the elements and causes the direction of the rotating magnetic moment to adjust to the direction of the inducing magnetic field. Anisotropic energy appears because of the interaction between atoms and crystal structure, and causes the direction of the magnetic moment of the elements to tend to be parallel to the crystal orientation of the MNPs. Exchange and magnetostatic energies appear as the consequences of the interaction between the magnetic moments of neighboring constituent elements. The existence of exchange interaction causes the direction of the magnetic moment of adjacent elements tends to be parallel to each other (Schrefl et al., 2006).
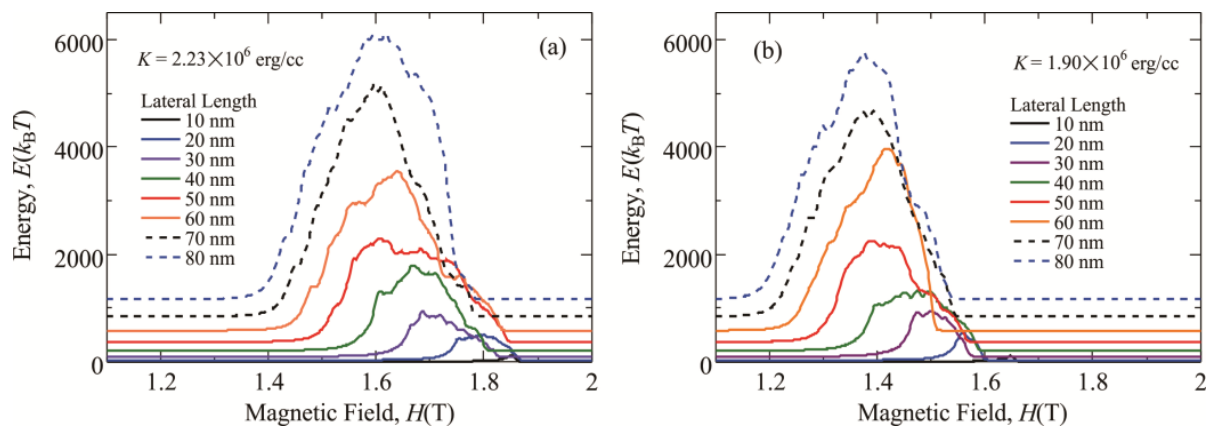

Figure 3. Energy of NPM Cobalt-ferrite for various sizes with the magnetic anisotropy constant as large as (a). $2.23 \times 10^{6} \mathrm{erg} / \mathrm{cc}$, dan (b). $1.90 \times 10^{6} \mathrm{erg} / \mathrm{cc}$. 
While being induced by a magnetic field, the total energy of MNPs is determined by the magnetic moment polarization of its elements. The total energy adjustment during the magnetic fiel induction is shown in Figure 3. In this process, barrier energy was formed, which separated the two minimum states. The first minimum state is related to the stable circumstance of the initial magnetization, whereas the second minimum state is related to the stable circumstance of the magnetization of the MNPs when it had been saturated due to the guidance of the external magnetic field. If the surface lateral length of the MNPs is larger, then the peak of this barrier shifts towards the lower field. In addition, the height of the barrier energy was also different for each surface size. This barrier represents the thermal stability of a material. Materials with low barrier easily lose its magnetism.

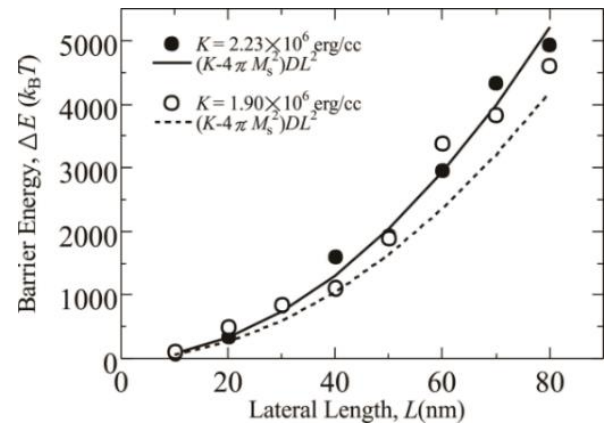

Figure 4. Size dependence of barrier energy.

The dependence of the barrier energy on the surface lateral length is presented in Figure 4. The larger surface area of the MNPs is, the higher barrier energy is. In this study, the dependence of the barrier energy on the MNP size is in-line with the previous predictions which fit to the Equation 1 (Purnama, 2009; Xiang \& Takeuchi, 2003; Liu et al., 2009).

$$
\Delta E=\left(K-2 \pi M_{s}^{2}\right) D L^{2}
$$

The first term of the equation includes the magnetic anisotropy parameter. The effect of anisotropy on the magnitude of $\Delta E$ is observed in Figure 4. The barrier energy was also lower for MNPs with small anisotropy. This magnetic anisotropy energy is the most dominant contributor in most particles in the form of thin layers. This parameter is the key to the properties of a magnetic material, which is a manifestation of the interaction between electron spins and orbital moments (spin-orbit coupling). Therefore, modification of the electronic structure on the surface and interface will have an impact on the strength of the magnetic anisotropy. This modification can be done by optimizing the particle synthesis process. On the other side, the second term comes from the magnetostatic energy because of the interaction between the magnetic moments of the adjacent elements. Modifications in the surface size of MNP determine the geometry ratio and the intensity of the magnetostatic field produced (Szambolics et al., 2009; Zeng et al., 2002).

For Cobalt-ferrite MNP with a lateral length of $10 \mathrm{~nm}$, the magnitude of the barrier energy can be compared to $k_{\mathrm{B}} T$. This indicates that in the absence of magnetic field, the magnetic strength of Cobalt-ferrite MNPs with $L=10 \mathrm{~nm}$ is relatively weak and will 
even disappear completely at a certain temperature. The loss of MNPs' magnetic properties when it is not exposed to an external magnetic field is a key factor in the drug delivery system because the agglomeration process can be prevented (Arruebo et al., 2007).

The magnetization orientation of Cobalt-ferrite MNP while it was being induced by an external magnetic field is shown in Figure 5. MNPs' magnetization which was initially saturated in the $x(+)$ direction, gradually transformed and reversed towards its stable state, along with the increase in the intensity of the inducing magnetic field. For two different anisotropic values, the particle size greatly influenced the shape of the magnetization curve. The larger surface size of MNPs is, the greater slope of the magnetization curve is. This result is in line with the research previously conducted by Anderson (Andersson, 2013).
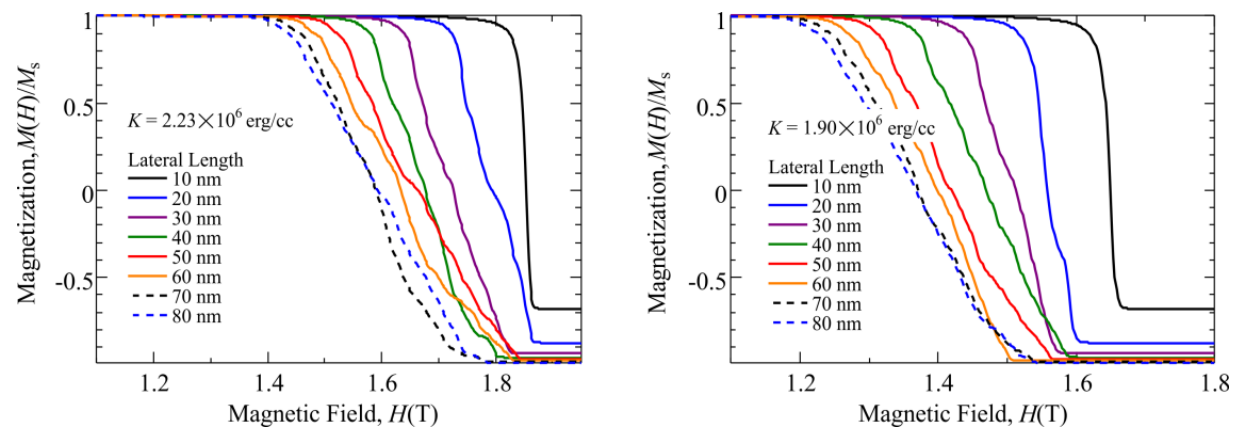

(a)
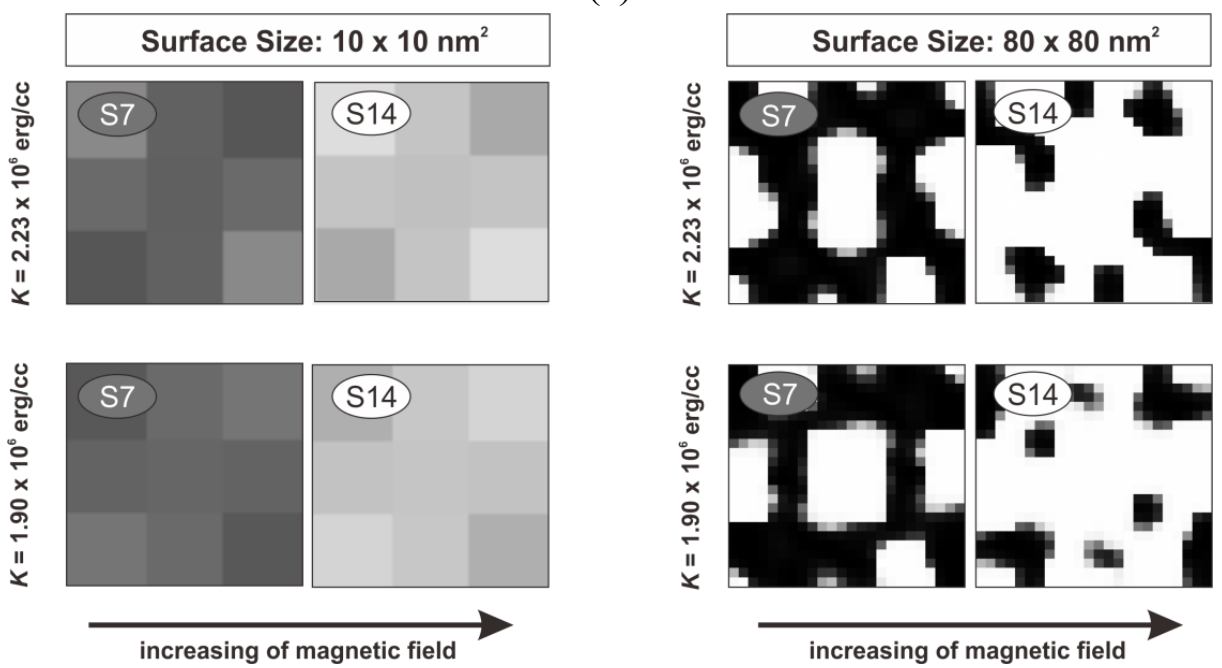

(b)

Figure 5. (a). Magnetization curve of NPM Cobalt-ferrite for various sizes, (b). Micro structure visualization.

The magnetization reversal mechanism of Cobalt-ferrite MNP with different surface sizes is presented in the form of micrograph in Figure 5(b). Black color represents the magnetic polarization of the elements in the $x$-axis direction $(+)$ (initial direction), white color represents the opposite direction (in the direction of the inducing field), whereas gray color represents the polarization in the other direction. Both code S7 and code S14 represent the stages during the magnetization reversal. This magnetization reversal 
mode of MNPs was strongly influenced by the particle size. For large particles $(L \geq 20$ $\mathrm{nm}$ ), the magnetization reversal transpired in a non-uniform mode (Fischbacher et al., 2018) with the formation of domain walls. On the other side, for small particles ( $L=10$ $\mathrm{nm}$ ), the reversal took place more uniformly through a spin rotation mechanism (Issa et al., 2013). The conversion in these reversal processes are related to the weakening of interactions between elements as a result of reducing the number of adjacent elements interacting with each other. This indicates that in small particles $(L=10 \mathrm{~nm})$, a single domain starts to be formed. This size is in line with the experiment conducted by Xu et al., in which with the thermal decomposition of a metal-organic salt method, they successfully synthesized a single-domain Cobalt-ferrite MNP with a size of $9 \mathrm{~nm}, 13$ $\mathrm{nm}$, and $16 \mathrm{~nm}$ (Xu et al., 2016).

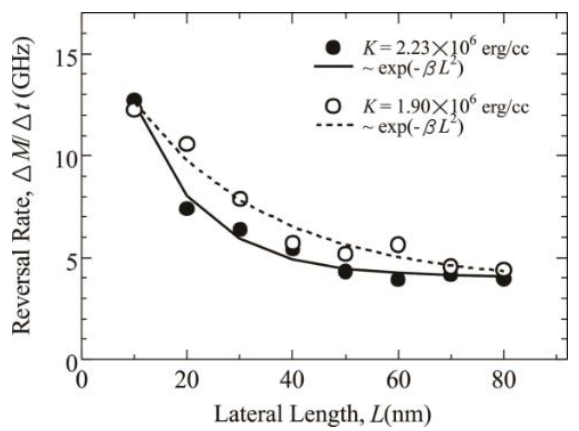

Figure 6. Surface size dependence of reversal rate.

Measurement of reversal time is highly important in studying the magnetization behavior of nano-sized magnetic particles (Moon et al., 2018). The lateral size dependence of magnetization rate for nano-Cobalt-ferrite is presented in Figure 6. The observed rate is in the $\mathrm{GHz}$ order (Moon et al., 2018). The magnetization reversal rate is strongly depending on the particle size. A slight modification in the size will have a major impact on the stability of the magnetization direction (Majetich, 1999). These simulations reveal that the magnetization reversal rate of Cobalt-ferrite MNP is sensitive to its size according to the exponential function previously addressed by Arrhenius-Neel:

$$
f \sim \exp \left(-\frac{\Delta E}{k_{\mathrm{B}} T}\right)
$$

where the magnetic moment reversal rate, $f$, is determined by the magnitude of the barrier energy between two stable magnetic states (Majetich \& Jin, 1999; Stier et al., 2018; Chantrell, 1988). Referring to Equation 1, that the barrier energy is proportional to the particle volume $\left(\Delta E \sim D L^{2}\right)$, the exponential decrease of the magnetization reversal rate against the surface area of Cobalt-ferrite MNP can be expressed in the following relation:

$$
\frac{\Delta M}{\Delta t} \sim \exp \left(-\beta L^{2}\right)
$$

with $\beta$ is a constant that depends on the particle thickness, temperature, and magnetic properties of a material, such as magnetic anisotropy and magnetic saturation. In the 
prospect of its application, the use of $10 \mathrm{~nm}$ Cobalt-ferrite MNP, which has a reversal rate of up to $\sim 12 \mathrm{GHz}$, is projected to be considerably useful in improving the sensors performance which based on the magnetic relaxation.

The nucleation field as a function of the surface size for Cobalt-ferrite MNP is shown in Fig.7(a). The nucleation field strengthened as the particle size decreased. This result corresponds to the research that was previously conducted by Schmidts and Kronmüller. In the study, an increase in the intensity of the nucleation field was also observed due to the size of the reduced particles (Chmidts \& Kronmüller, 1991). On the other hand, the magnitude of the coercive field of Cobalt-ferrite MNP on various surface sizes is shown in Figure 7(b). In the range of lateral lengths from $10 \mathrm{~nm}$ to $80 \mathrm{~nm}$, the intensity of the coercive field of Cobalt-ferrite MNP was in the range of 5-20 kOe. The magnitude of the coercive field in this simulation is quite realistic compared to Limaye et al.'s research which found that the coercivity of Cobalt-ferrite MNP of $\sim 20 \mathrm{~nm}$ was in the range of $930-9470$ Oe with the annealing temperature variations reduced from $1473 \mathrm{~K}$ to $373 \mathrm{~K}$ (Limaye et al., 2009). In MNPs with a narrowed surface, the coercive field strengthened. This result confirms other studies. In the microstructure simulation study carried out by previous researchers, a strengthening of the coercivity towards the decreasing particle dimensions was also observed (Hadjipanayis \& Kim, 1988; Durst \& Kronmüller, 1987; Bance et al., 2014). This result was also supported by experimental data from several other studies. In his experiments on Cobalt nanowires, Zeng et al. observed that in the range of the decreasing diameter of nanowires from 20 to $10 \mathrm{~nm}$, there was a strengthening of coercive field (Zeng et al., 2002). The same pattern was also observed experimentally by (Fukada et al., 2012), (Uestuener et al., 2006) and (Ramesh et al., 1988). There is no specific function that correlates particle size with magnitude of the coercive field. This is due to the large number of parameters, in addition to the particle size, which affect the microstructure of the particles, including the time and temperature of the sintering process, and the composition of the particle elements (Schmidts \& Kronmüller, 1991). Referring to Figure 5, the increase in coercivity along with the reduction of the particle surface size can be attributed to the transformations of magnetization reversal modes from the domain wall nucleation to the rotation of magnetic moment (Issa et al., 2013) as the effects of the reduced interaction of the adjacent elements.
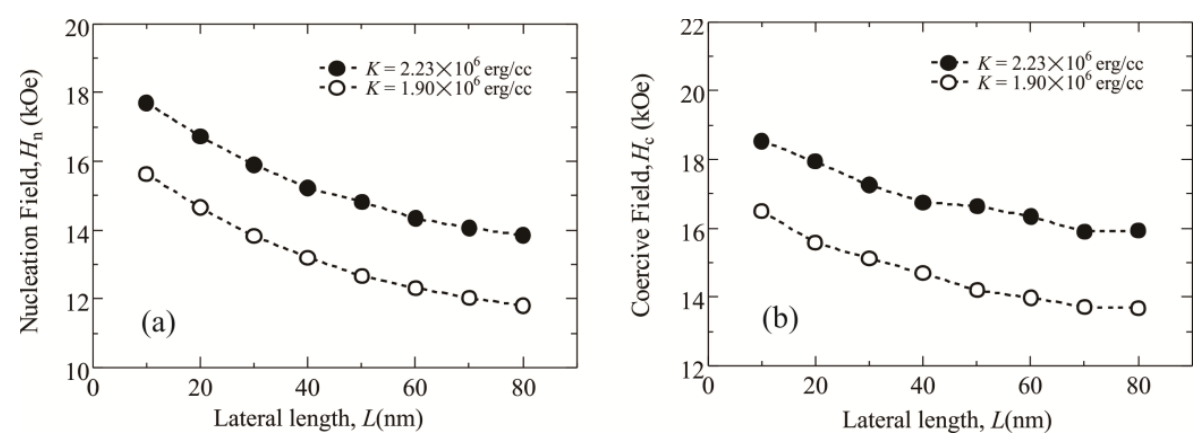

Figure 7. Size dependence of (a). Nucleation field, dan (b). Coercive field. 
In addition to the surface size impression in Figure 7, it was also observed that the magnetic anisotropy of the MNPs greatly influenced the magnitude of the nucleation and coercive fields. These two types of fields are interrelated (Fischbacher et al., 2018), where the magnitude of the nucleation field will never exceed the coercive field. Both of these fields had the same response to the material anisotropy. The material anisotropy induced an increase in the coercive field (Issa et al., 2013) as well as the nucleation field (Zhao et al., 2017). The result of this increase was the larger of the hysteresis curve, so that the dissipated energy was also getting higher (Salas-Solis et al., 2004). The high coercive field of Cobalt-ferrite MNP at $L=10 \mathrm{~nm}$ indicates that the superparamagnetic phenomenon had not been realized yet even though a single domain had been formed. This is confirmed by Kafrouni and Savadogo who stated that the maximum size of Cobalt-ferrite MNP is still superparamagnetic at $5 \mathrm{~nm}$ (Kafrouni \& Savadogo, 2016).

\section{Conclusion}

Micromagnetic simulation study was carried out on the magnetic properties of Cobalt-ferrite magnetic nanoparticles in its relation to modifications in the size of a square surface. It was observed that changes in particle surface size had a significant impact on various magnetic properties of Cobalt-ferrite MNP, which are the magnitude of barrier energy, coercive and nucleation fields, magnetization reversal rate process, magnetization curve profile, and magnetization reversal mode.

From the results of these micromagnetic simulations, four characteristics of Cobaltferrite MNP were observed with a lateral length of $10 \mathrm{~nm}$. First, magnetism will lost easily due to the heat fluctuations when it was not exposed to the field. Second, a single domain was formed, so the magnetization reversal process during the induction with the external magnetic field took place in rotation. Third, magnetization reversal took place with a high frequency. Fourth, high coercivity was observed, which indicated that Cobalt-ferrite MNP of that size had not been in the superparamagnetic phase. The high coercivity can be correlated with the weakening of the interaction energy between elements as a result of reducing the number of adjacent elements interacting with each other.

\section{References}

Akbarzadeh, M. A., Samiei, and S. Davaran. (2012). "Magnetic nanoparticles: preparation, physical properties, and applications in biomedicine," Nanoscale Res Lett, vol. 7, no. 1, p. 144, doi: 10.1186/1556-276X-7-144.

Amiri S. \& H. Shokrollahi. (2013). The role of cobalt ferrite magnetic nanoparticles in medical science," Materials Science and Engineering: C, vol. 33, no. 1, pp. 18, Jan., doi: 10.1016/j.msec.2012.09.003.

Andersson, M. (2013) Modeling and characterization of magnetic nanoparticles intended for cancer treatment.

Arruebo, M. R. Fernández-Pacheco, M. R. Ibarra, and J. Santamaría, (2007), "Magnetic nanoparticles for drug delivery," Nano Today, vol. 2, no. 3, pp. 22-32, doi: 10.1016/S1748-0132(07)70084-1. 
Azizah, U. M. N. M. B. Jessajas, C. Handoyo, and N. A. Wibowo, (2017), "Characteristic of Nano-barium-ferrite as Recording Media Using HAMR Technology," Chiang Mai J. Sci., vol. 44, no. 4, pp. 1669-1675.

Bance S. et al., (2014), "Grain-size dependent demagnetizing factors in permanent magnets," Journal of Applied Physics, vol. 116, no. 23, p. 233903, doi: 10.1063/1.4904854.

Bance, S. J. Fischbacher, A. Kovacs, H. Oezelt, F. Reichel, and T. Schrefl. (2015). "Thermal Activation in Permanent Magnets," JOM, vol. 67, no. 6, pp. 13501356, doi: 10.1007/s11837-015-1415-7.

Bedanta, S. A. Barman, W. Kleemann, O. Petracic, and T. Seki. (2013). "Magnetic Nanoparticles: A Subject for Both Fundamental Research and Applications," Journal of Nanomaterials, vol. 2013, no. 952540 doi: 10.1155/2013/952540.

Chantrell, R. W. G. N. Coverdale, and K. O, (1988), textquotesingleGrady, "Time dependence and rate dependence of the coercivity of particulate recording media," J. Phys. D: Appl. Phys., vol. 21, no. 9, pp. 1469-1471, doi: 10.1088/0022-3727/21/9/026.

Durst K.-D. \& H. Kronmüller, (1987), "The coercive field of sintered and melt-spun NdFeB magnets," Journal of Magnetism and Magnetic Materials, vol. 68, no. 1, pp. 63-75, doi: 10.1016/0304-8853(87)90097-7.

Fischbacher, J. et al. (2018). "Micromagnetics of rare-earth efficient permanent magnets," J. Phys. D: Appl. Phys., vol. 51, no. 19, p. 193002, doi: 10.1088/1361-6463/aab7d1.

Fukada T. et al., (2012), "Evaluation of the Microstructural Contribution to the Coercivity of Fine-Grained Nd-Fe-B Sintered Magnets," Materials Transactions, vol. 53, no. 11, pp. 1967-1971, doi: 10.2320/matertrans. MAW201207.

Ganachari S. V. et al., (2017). "Synthesis Techniques for Preparation of Nanomaterials," in Handbook of Ecomaterials, L. M. T. Martínez, O. V. Kharissova, and B. I. Kharisov, Eds. Cham: Springer International Publishing. pp. 1-21.

Ghazanfari, M. R. M. Kashefi, S. F. Shams, and M. R. Jaafari. (2016). "Perspective of Fe3O4 Nanoparticles Role in Biomedical Applications," Biochemistry Research International, no. 7840161, doi: 10.1155/2016/7840161.

Guo, T. et al. (2018). "The Recent Advances of Magnetic Nanoparticles in Medicine," Journal of Nanomaterials, vol. 2018, no. 7805147. doi: $10.1155 / 2018 / 7805147$.

Hadjipanayis, G. C. \& A. Kim, (1988) "Domain wall pinning versus nucleation of reversed domains in R-Fe-B magnets (invited)," Journal of Applied Physics, vol. 63 , no. 8 , pp. 3310-3315, doi: 10.1063/1.340821.

Houshiar, M. F. Zebhi, Z. J. Razi, A. Alidoust, and Z. Askari. (2014). "Synthesis of cobalt ferrite (CoFe2O4) nanoparticles using combustion, coprecipitation, and precipitation methods: A comparison study of size, structural, and magnetic properties," Journal of Magnetism and Magnetic Materials, vol. 371, pp. 4348, doi: 10.1016/j.jmmm.2014.06.059. 
Issa, B. I. M. Obaidat, B. A. Albiss, and Y. Haik, (2013), "Magnetic Nanoparticles: Surface Effects and Properties Related to Biomedicine Applications," Int J Mol Sci, vol. 14, no. 11, pp. 21266-21305, doi: 10.3390/ijms141121266.

Kafrouni L. \& O. Savadogo, (2016), "Recent progress on magnetic nanoparticles for magnetic hyperthermia," Prog Biomater, vol. 5, no. 3, pp. 147-160, doi: 10.1007/s40204-016-0054-6.

Limaye, M. V. et al. (2009). "High Coercivity of Oleic Acid Capped CoFe2O4 Nanoparticles at Room Temperature," J. Phys. Chem. B, vol. 113, no. 27, pp. 9070-9076. doi: 10.1021/jp810975v.

Liu, J. P. E. Fullerton, O. Gutfleisch, and D. J. Sellmyer, (2009), Eds., Nanoscale Magnetic Materials and Applications. Springer US.

Majetich, S. A. \& Y. Jin, (1999), "Magnetization Directions of Individual Nanoparticles," Science, vol. 284, no. 5413, pp. 470-473, doi: 10.1126/science.284.5413.470.

Mohamed, R. M. M. M. Rashad, F. A. Haraz, and W. Sigmund. (2010). "Structure and magnetic properties of nanocrystalline cobalt ferrite powders synthesized using organic acid precursor method," Journal of Magnetism and Magnetic Materials, vol. 322, no. 14, pp. 2058-2064, doi: 10.1016/j.jmmm.2010.01.034.

Moon, J.-H. T. Y. Lee, and C.-Y. You, (2018), "Relation between switching time distribution and damping constant in magnetic nanostructure," Sci Rep, vol. 8, no. 1, pp. 1-8, Sep. doi: 10.1038/s41598-018-31299-4.

Purnama, B. (2009). “Thermally assisted magnetization reversal in perpendicularly magnetized thin film,” PhD Thesis, 九州大学.

Purnama, B. M. Koga, Y. Nozaki, and K. Matsuyama, (2009), "Stochastic simulation of thermally assisted magnetization reversal in sub-100nm dots with perpendicular anisotropy," Journal of Magnetism and Magnetic Materials, vol. 321, no. 9, pp. 1325-1330, doi: 10.1016/j.jmmm.2008.12.003.

Purnama, B. R. Rahmawati, A. T. Wijayanta, and Suharyana. (2015). "Dependence of Structural and Magnetic Properties on Annealing Times in Co-precipitated Cobalt Ferrite Nanoparticles," Journal of Magnetics, vol. 20, no. 3, pp. 207210.

Purnama, B. Y. Nozaki, and K. Matsuyama, (2007), "Micromagnetic simulation of thermally assisted magnetization reversal in magnetic nanodots with perpendicular anisotropy," Journal of Magnetism and Magnetic Materials, vol. 310, no. 2, Part 3, pp. 2683-2685, doi: 10.1016/j.jmmm.2006.10.988.

Ramesh, R. G. Thomas, and B. M. Ma, (1988), "Magnetization reversal in nucleation controlled magnets. II. Effect of grain size and size distribution on intrinsic coercivity of Fe-Nd-B magnets," Journal of Applied Physics, vol. 64, no. 11, pp. 6416-6423, doi: 10.1063/1.342055.

Richter H. J. \& S. D. Harkness, (2006), "Media for Magnetic Recording Beyond 100 Gbit/in.2," MRS Bulletin, vol. 31, no. 5, pp. 384-388, doi: $10.1557 / \mathrm{mrs} 2006.98$.

Rondinone, A. J. A. C. S. Samia, and Z. J. Zhang. (2000). "Characterizing the magnetic 
anisotropy constant of spinel cobalt ferrite nanoparticles," Appl. Phys. Lett., vol. 76, no. 24, pp. 3624-3626, doi: 10.1063/1.126727.

Salas-Solis, M. C. F. Aguilera-Granja, J. Cartes, S. Contreras, and E. E. Vogel, (2004), "Axial anisotropic effects in hysteresis of $\pm \mathrm{J}$ Ising lattices," Physical Review B, vol. 70, p. 064404, doi: 10.1103/PhysRevB.70.064404.

Schmidts H. F. \& H. Kronmüller, (1991), "Size dependence of the nucleation field of rectangular ferromagnetic parallelepipeds," Journal of Magnetism and Magnetic Materials, vol. 94, no. 1, pp. 220-234, Mar., doi: 10.1016/03048853(91)90131-S.

Schrefl, T. J. Fidler, D. Suess, W. Scholz, and V. Tsiantos, (2006), "Micromagnetic Simulation of Dynamic and Thermal Effects," in Handbook of Advanced Magnetic Materials, Y. Liu, D. J. Sellmyer, and D. Shindo, Eds. Boston, MA: Springer US, pp. 128-146.

Shir, C. C. (1978). "Computations of the micromagnetic dynamics in domain walls," Journal of Applied Physics, vol. 49, no. 3, pp. 1841-1843, doi: $10.1063 / 1.324832$.

Srinivasan, S. Y. K. M. Paknikar, D. Bodas, and V. Gajbhiye. (2018). "Applications of cobalt ferrite nanoparticles in biomedical nanotechnology," Nanomedicine (Lond), vol. 13, no. 10, pp. 1221-1238, doi: 10.2217/nnm-2017-0379.

Stier, M. A. Neumann, A. Philippi-Kobs, H. P. Oepen, and M. Thorwart, (2018), "Implications of a temperature-dependent magnetic anisotropy for superparamagnetic switching," Journal of Magnetism and Magnetic Materials, vol. 447, pp. 96-100, doi: 10.1016/j.jmmm.2017.09.068.

Suess et al., (2002), "Time resolved micromagnetics using a preconditioned time integration method," Journal of Magnetism and Magnetic Materials, vol. 248, no. 2, pp. 298-311, Jul. 2002, doi: 10.1016/S0304-8853(02)00341-4.

Szambolics, H. J.-Ch. Toussaint, A. Marty, I. M. Miron, and L. D. Buda-Prejbeanu, (2009), "Domain wall motion in ferromagnetic systems with perpendicular magnetization," Journal of Magnetism and Magnetic Materials, vol. 321, no. 13, pp. 1912-1918, doi: 10.1016/j.jmmm.2008.12.011.

Uestuener, K. M. Katter, and W. Rodewald, (2006), "Dependence of the Mean Grain Size and Coercivity of Sintered Nd-Fe-B Magnets on the Initial Powder Particle Size," in 2006 IEEE International Magnetics Conference (INTERMAG), pp. 228-228, doi: 10.1109/INTMAG.2006.375810.

Wibowo, N. A. C. Handoyo, and L. R. Sasongko, (2019), "Thermally activated magnetic switching mode for various thicknesses of perpendicularly ferromagnetic nano-dot," Nanoscience and Nanotechnology - Asia, vol. 9, no. 2, pp. 259-266, 2019, doi: 10.2174/2210681208666180507101809.

Wibowo, N. A. D. B. Nugroho, and C. A. Riyanto, (2019), "Performance of magnetic switching at the recording temperature in perpendicularly magnetized nanodots," Journal of Magnetics, vol. 24, no. 1, pp. 17-23, doi: 10.4283/JMAG.2019.24.1.017.

Wibowo, N. A. F. S. Rondonuwu, and B. Purnama, (2014), "Low Writing Field on Perpendicular Nano-ferromagnetic," Journal of Magnetics, vol. 19, no. 3, pp. 
237-240, doi: 10.4283/JMAG.2014.19.3.237.

Xiang, X.-D. \& I. Takeuchi, (2003), Combinatorial Materials Synthesis, 1st ed. CRC Press.

Xu, S. Y. Ma, B. Geng, X. Sun, and M. Wang, (2016), "The remanence ratio in $\mathrm{CoFe} 2 \mathrm{O} 4$ nanoparticles with approximate single-domain sizes," Nanoscale Res Lett, vol. 11, no. 1, p. 471, doi: 10.1186/s11671-016-1691-3.

Zeng, H. R. Skomski, L. Menon, Y. Liu, S. Bandyopadhyay, and D. J. Sellmyer, (2002), "Structure and magnetic properties of ferromagnetic nanowires in selfassembled arrays," Physical Review B, vol. 65, p. 134426, doi: 10.1103/PhysRevB.65.134426.

Zhao, Q. J. Chen, J. Wang, X. Zhang, G. Zhao, and Q. Ma, (2017), "The effect of interface anisotropy on demagnetization progress in perpendicularly oriented hard/soft exchange-coupled multilayers," Sci Rep, vol. 7, no. 1, pp. 1-11, doi: 10.1038/s41598-017-03169-y. 